\title{
Biomedical Arrhythmia Heart Diseases Classification Based on Artificial Neural Network and Machine Learning Approach
}

\author{
Stalin Subbiah $^{1 *}$, Suresh Subramanian ${ }^{2}$ \\ ${ }^{1}$ Lecturer, Electrical \& Electronics Division, Bahrain Training Institute, Kingdom of Bahrain. \\ ${ }^{2}$ Assistant Professor, College of Information Technology, Department of Multimedia Science, Ahlia University, Kingdom of Bahrain. \\ *Corresponding author E-mail: phdstalin@gmail.com
}

\begin{abstract}
In present day, several types of developments are carried toward the medical application. There has been increased improvement in the processing of ECG signals. The accurate detection of ECG signals with the help of detection of P, Q, R and S waveform. However these waveforms are suffered from some disturbances like noise. Initially denoising the ECG signal using filters and detect the PQRS waveforms. Four filters are carried out to remove the ECG noises that are Median, Gaussian, FIR and Butterworth filter. ECG signal is analyzed or classify using Extreme Learning Machine (ELM) and it compared with Support Vector Machine (SVM) and Back Propagation Neural Network (BPNN). The paper classifies the ECG signal into two classes, Normal and Abnormal. ECG waveform is detected and analyzed using the 48 records of the MIT-BIH arrhythmia database. Denoising results are evaluated using MSE, RMSE, PSNR, NAE and NCC. The classifier performance is measured in terms of Sensitivity (Se), Positive Predictivity (PP) and Specificity (SP).
\end{abstract}

Keywords: Electrocardiogram, extreme learning machine, support vector machine, back prorogation neural network, MIT-BIH arrhythmia database.

\section{Introduction}

ECG is abbreviated as Electrocardiogram it is used to represent the electrical activity of the heart. It means that it showing the heart muscle contraction and relaxation. Electrocardiography is another term is used to give or provide the heart condition. Analysis of the ECG waveform is used to identify the heart normal and abnormalities. Basically ECG waveform has some basic waves they are $\mathrm{P}, \mathrm{Q}, \mathrm{R}, \mathrm{S}, \mathrm{T}$ and $\mathrm{U}$ and it is shown in figure1. These waveforms are used to analyses the heart condition. The main important one in ECG is depolarization and repolarization. Atrial depolarization is represented by $\mathrm{P}$ waveform and ventricular depolarization is represented by QRS complex waveform and then repolarization of the ventricle is represented by $\mathrm{T}$ waveform [1].

There are several existing methods for ECG waveform analysis and provides the system with accuracy and sensitivity. These methods are based on various techniques that are wavelet, RBF Neural network, fuzzy with clustering technique, machine learning of SOM and autoregressive modeling [2, 3, 4, 5 and 6]. There are several techniques are analyzed the ECG signal and it is described in [7-10].

Combination of recurrent neural networks and eigenvectors are used to analyze the ECG signal [11]. Here four type of beats are analyzed they are normal beat, congestive heart failure beat, ventricular tachyarrhythmia beat, atrial fibrillation beat collected from Physio bank database. Another combination of novel method for analyzed the ECG signal using particle swarm optimization and radial basis function neural network [12]. Here author analyzed the six types of beats that are Normal Beat, Premature Ventricular Contraction, Fusion of Ventricular and Normal Beat, Atrial Premature Beat, Right Bundle Branch Block Beat and Fusion of Paced and Normal Beat. These beats are collected from the MIT-BIH arrhythmia database, four types of morphological features are extracted from these beats and it is used for classification. Radial basis function neural network is used for classification and it is evolved using particle swarm optimization technique. Apart from MIT-BIH database, ECG signal is collected from European ST-T database and it is described in [13]. In this work author classified that the single ECG beat as normal or ischemic based on supervised neural network.

In [14] author introduce the ECG beat segmentation technique and this method is consists of two stages, that are signal processing and ECG detector. In signal processing, noises in the ECG signals are removed using wavelet and low pass filter approaches. ECG detector is the second stage, here extracted the ECG beats by apply one adaptive threshold value.

Several noises are affected the ECG signal and the respective noises are produced by motion artifacts and some instrument error. In recent year ECG signal noise is removed by using sparse derivatives [15].

The paper is organized as follows. In Section 2, describes about some related work. In Section 3, the proposed ECG algorithm, In Section 4, we describe a complete automatic QRS detection system to show how the proposed ECG enhancement method helps increase the overall detection accuracy. The system is applied to the whole MIT-BIH Arrhythmia database and the performance is compared to some other state-of-art methods.

\section{Related Work}

In [17] ECG signal is analyzed using cluster analysis method, this technique consists of three major steps they are extracting the QRS waveform, second stage is selecting qualitative features and the third stage is determining heartbeat case. This method analyses and classified the normal and abnormal heartbeat. 
In [16] ECG signal noise reductions are carried through the novel approach of combination of discrete wavelet transform and artificial neural network. The work of the wavelet transform is decomposes the ECG signal and remove the noise then the second stage of the artificial neural network is implemented the inverse transform and adaptive filtering for remove the remaining noise. In [18], author presented a KNN algorithm as a classifier for detection of QRS complex. Explore the method by using standard CSE ECG database and MIT-BIH Arrhythmia database. QRS complex detection depend on: (i) digital filter is used to remove the noise; (ii) slope of the ECG signal is used for QRS complex detection.

In [19], author has presented an algorithm for detection of R-peak based on: (i) location of QRS region by using sorting and thresholding of the square doubled ECG signal, (ii) relative magnitude comparison of QRS regions and (iii) to get the accurate detection of $\mathrm{R}$ weak using RR interval and these are all analyzed by using PTB diagnostic ECG database.

In [20], authors have presented the multi-resolution wavelet transform and Artificial Neural Network classifier for ECG abnormalities detection. It classifies the ECG signal into two classes that are normal and abnormal class. Classifications have taken in three types: (i) Back Propagation Network (BPNN), (ii) Feed Forward Network (FFN) and (iii) Multilayered Perceptron (MLP).

\section{Methodology}

Block diagram of proposed framework is shown in figure 1. The figure provides the whole framework is divided into three stages that are pre-processing, peak detection, and heart beat classification. Input signal are picked from MIT-BIH arrhythmia database and is given to pre-processing. Pre-processing is used to denoise the ECG signal and this process is given as an input to the next stage. The next stage of the proposed framework is peak detection and QRS complex, before QRS complex ECG signal is decomposed used Wavelet transform. It is one of the powerful techniques in biomedical signal processing. QRS complex features are given as input as a classifier and identified the heartbeat as a normal and abnormal. ECG signal are classified by BPNN, SVM and ELM. Compared to BPNN and SVM, ELM provides the simpler implementation, learning speed is fast and provide better performance.Database is one of the most important tasks for proposed work. Here ECG signals are collected from MIT-BIH arrhythmia. This database contains 48 records, and these records are illustrated by a text headed file (.hea), a binary file (.dat) and a binary annotation file (.atr). The header files describe the detailed information such as number of samples, sampling frequency, format of ECG signal, type of ECG leads and number of ECG leads, patient's history and the detailed clinical information. In binary annotation file, the signal is stored in 212 format which means each one sample necessitates number of leads times 12 bits to be stored and the binary annotation file consists of beat annotations [21].

\section{Preprocessing}

Preprocessing is one of the important tasks in signal and image processing.

This is the first step for proposed ECG classification. The work of the preprocessing is to eliminate the noise in the input ECG signal using various filters approaches. Proposed work handled median filter, FIR filter, Gaussian filter and Butterworth filter for noise removal in ECG signal [22].Preprocess result is used to get the better efficiency of the ECG signal. Peak detection efficiency is increased due to preprocessing work. Various filters approaches are used to remove the noise of baseline wander, power interference, and instrumental error. Elimination of baseline wander is therefore needed in the ECG signal analysis to diminish the irregularities in beat morphology. Finally median filter provides the better result and preserve the edges.

\section{Peak Detection}

ECG signal is extracted using wavelet decomposition; this is done by Daubechies6 (DB6) multi resolution wavelet. Figure 3 shows the wavelet decomposition. In that figure $\mathrm{C}$ and $\mathrm{L}$ denotes the wavelet decomposition vector and their wavelet bookkeeping vector. Then reconstruct the signal based on wavelet decomposition C and L. ECG signal is decomposed up to eight levels. This type of decomposition is used to extract the features and helpful for the selection of $\mathrm{P}, \mathrm{Q}, \mathrm{R}$ and $\mathrm{S}$ wave. Then all the peaks are identified by minimum and maximum value. Figure 3 illustrates the algorithm for QRS complex. Using QRS complex, in this paper find out the heart beat and these beats are given to the classification to deliver the classify output as normal and abnormal.

\section{Classification}

\section{Back Propagation Neural Network}

Given a finite length input patterns $X_{1}(k), X_{2}(k), \ldots, X_{n}(k) \in$ $\mathrm{R},(1 \leq \mathrm{k} \leq \mathrm{k})$ and the desired patterns $\mathrm{X}_{1}(\mathrm{k}), \mathrm{X}_{2}(\mathrm{k}), \ldots, \mathrm{X}_{\mathrm{m}}(\mathrm{k}) \in$ R.

Step 1: Select the total number of layers $\mathrm{M}$, the number $n_{i}(i=$ $1,2, \ldots, M-1)$ of the neurons in each hidden layer, and an error tolerance parameter $\varepsilon>0$.

Step 2: Randomly select the initial values of the weight vectors $W_{a j}^{(i)}$ for $i=1,2, \ldots, n_{i}$.

Step 3: Initialization:

$$
W_{a j}^{(i)} \leftarrow W_{a j}^{(i)}(0), E \leftarrow 0, k \leftarrow 1
$$

Step 4: Calculate the neural outputs

$$
\left\{\begin{array}{c}
s_{j}^{(i)}=\left(W_{a j}^{(i)}\right)^{T} X_{a}^{(i-1)} \\
X_{j}^{(i)}=\sigma\left(S_{j}^{(i)}\right)
\end{array}\right.
$$

For $i=1,2, \ldots, M$ and $j=1,2, \ldots, n_{i}$

Step 5: Calculate the output error

$$
e_{j}=d_{j}-X_{j}^{(M)}
$$

Step 6: Calculate the output deltas

$$
\delta_{j}^{(M)}=e_{j} \sigma^{\prime}\left(s_{j}^{(M)}\right)
$$

Step 7: Recursively calculate the propagation errors of the hidden neurons

$$
e_{j}^{(i)}=\sum_{l=1}^{n_{i}+1} \delta_{l}^{(i+1)} W_{l j}^{(i+1)}
$$

From the layer $M-1, M-2, \ldots$, to layer 1 .

Step 8: Recursively calculate the hidden neural delta values.

Step 9: Update weight vectors

$$
\delta_{j}^{(i)}=e_{j} \sigma^{\prime}\left(s_{j}^{(i)}\right)
$$

$$
W_{a j}^{(i)}=W_{a j}^{(i)}+\boldsymbol{\eta} \boldsymbol{\delta}_{\boldsymbol{j}}^{(i)} \boldsymbol{X}_{\boldsymbol{a}}^{(i-1)}
$$

Step 10: Calculate the error function

$$
E=E+\frac{1}{k} \sum_{j=1}^{m} e_{j}^{2}
$$

Step 11: if $\mathrm{k}=\mathrm{K}$ then go to step 12; otherwise, $k \leftarrow k+1$ and go to step 4.

Step 12: if $E \leq \varepsilon$ then go to step 13; otherwise go to step 3 .

Step 13: learning is completed. Output the weights

\section{Support Vector Machine}

Support Vector Machine (SVM) is one of the learning system and it is used mainly in classification. It was developed in the year of 
1998 by Vapnik and it is one of the most techniques to solve the supervised classification problem. In essence, SVM classifiers maximize the margin between training data and the decision boundary (optimal separating hyperplane), which can be formulated as a quadratic optimization problem in a feature space. The subset of patterns those are closest to the decision boundary are called support vectors.

Consider a set of training examples $\left(x_{1}, y_{1}\right), \ldots,\left(x_{l}, y_{l}\right)$, here input $x_{i} \in R^{N}$ and class labels $y_{i} \in\left\{R^{N}\right\}$ and class labels $y_{i} \in\{-1,+1\}$. For alinearly separable classification problem, the construction of a hyperplane is $w^{T} x+b=0$ so that the margin betweenthe hyperplane and the nearest point is maximized and can be posed as the following quadratic optimization problem:

$$
\min _{w} \frac{1}{2}\left(w^{T} w\right)
$$

Subject to $y_{i}\left(\left(w^{T} x_{i}\right)+b \geq 1\right)$, where $i=1, \ldots, l$

Equation 2 forces a rescaling on $(w, b)$ so that the point nearest to the hyperplane has a distance of $(1 /\|w\|)$ [23].

In many practical situations, a separating hyperplane does not exist. To allow the possibilities of violating (2), slack variables $\xi_{i}$ are introduced like

To get

$$
\xi_{i} \geq 0, \mathrm{i}=1, \ldots, \mathrm{l}
$$

$$
y_{i}\left(\left(w^{T} x_{i}\right)+b\right) \geq 1-\xi_{i}, i=1, \ldots, l
$$

The optimization problem now becomes as follows:

$$
\min _{w, \xi} \frac{1}{2}\left(w^{T} w\right)+C \sum_{i=1}^{l} \xi_{i}
$$

Subject to constraints ( 3 ) and (4). The $\mathrm{C}$ is a user defined constant. It is called regularizing parameter and determines the balance between the maximization of the margin and the minimization of the classification error.

By introducing Lagrange multipliers $\propto_{i}$ and using Karush-KuhnTucker theorem of optimization theory, thesolution is given by;

$$
w=\sum_{i=1}^{l} y_{i} \propto_{i} x_{i}
$$

Only a small fraction of the $\propto_{i}$ coefficients are nonzero. The corresponding pairs of $x_{i}$ entries are known as support vectorsand they fully define the decision boundary. All other training examples with corresponding zero $\propto_{i}$ values arenow rendered irrelevant and automatically satisfy constraint (4) with $\xi_{i}=0$.The hyperplane decision function for the vector $\mathrm{x}$ can be written as follows

$$
f(x)=\operatorname{sgn}\left[\sum_{i=1}^{l} y_{i} \propto_{i}\left(x^{T} x_{i}\right)+b\right]
$$

By replacing the inner product $\left(x^{T} x_{i}\right)$ with kernel function $K\left(x, x_{i}\right)$; the input data are mapped to a higher dimensional space [23]. It is then in this higher dimensional pace that a separating hyperplane is constructed to maximize the margin.

\section{Extreme Learning Machine}

Recently in [24] have developed a new learning algorithm called Extreme Learning Machine (ELM) for Single-hidden Layer Feed forward neural Networks(SLFNs). In this technique hidden node parameters are randomly chosen and fixed and then analytically determine the output weight of SLFNs [25]

\section{Single-Hidden Layer Feed Forward Neural Networks}

NHidden nodes are the output of an SLFN and this can be represented by

$$
f_{\widetilde{N}}(X)=\sum_{i=1}^{\widetilde{N}} \beta_{i} G\left(a_{i} b_{i} X\right) \text {, where } X \epsilon R^{n}, a_{i} \in R^{n}
$$

In this equation, the learning parameters of hidden nodes are given bya $_{\mathrm{i}} \mathrm{andb}_{\mathrm{i}}$, then $\beta_{\mathrm{i}}$ indicates the weight connecting the ith hidden node to the output node. Output of the ith hidden node with respect to the input $x$ is given byG $\left(a_{i} b_{i} X\right)$.

For additive hidden node with the activation function $g(x): R \rightarrow$ $R, G\left(a_{i} b_{i} X\right)$ is given by

$$
G\left(a_{i} b_{i} X\right)=g\left(a_{i} . X+b_{i}\right), \text { where } b_{i} \in R
$$

In the above equation $a_{i}$ is the weight vector connecting the input layer to the ith hidden node and $b_{i}$ is the bias of the ith hidden node and then $a_{i}$. $X$ indicates the inner product of vectors $a_{i}$ and $\mathrm{XinR}^{\mathrm{n}}$.For an $\mathrm{RBF}$ hidden node with an activation function $g(x): R \rightarrow R, G\left(a_{i} b_{i} X\right)$ is given by

$$
G\left(a_{i} b_{i} X\right)=g\left(b_{i}\left\|X-a_{i}\right\|, b_{i} \in R^{+}\right)
$$

In the above equation $a_{i}$ and $b_{i}$ are the center and impact factor of theith RBF node. $R^{+}$indicates the set of all positive real values.The RBF network is a special case of SLFN with RBF nodesin its hidden layer. Each RBF node has its own centroid andimpact factor and its output is given by a radiallysymmetric function of the distance between the input and the center.

\section{Extreme Learning Machine}

Learning algorithm use a finite number of input and outputs for training in supervised batch learning system. In this system consider $\mathrm{N}$ arbitrary distinct samples $\left(X_{i}, t_{i}\right) \in R^{n} \times R^{m}$, in this $X_{i}$ is an $n \times 1$ input vectors and $t_{i}$ is a $m \times 1$ target vector. If an SLFN with $\widetilde{N}$ hidden nodes can approximate these $\mathrm{N}$ samples with zero error, it then implies that there exist $\beta_{i}, a_{i}$ and $b_{i}$ such that

$$
f_{\widetilde{N}}\left(X_{j}\right)=\sum_{i=1}^{\widetilde{N}} \beta_{i} G\left(a_{i}, b_{i}, X_{j}\right)=t_{j}, j=1, \ldots, N
$$

The above equation can be written as

$$
H \beta=T
$$

Where

$$
\begin{gathered}
H\left(a_{1}, \ldots, a_{\widetilde{N}}, b_{1}, \ldots, b_{\widetilde{N}}, X_{1}, \ldots, X_{N}\right) \\
=\left[\begin{array}{ccc}
G\left(a_{1}, b_{1}, X_{1}\right) & \ldots & G\left(a_{\widetilde{N}}, b_{\widetilde{N}}, X_{1}\right) \\
\vdots & \ldots & \vdots \\
G\left(a_{1}, b_{1}, X_{N}\right) & \ldots & G\left(a_{\widetilde{N}}, b_{\widetilde{N}}, X_{N}\right)
\end{array}\right]_{N \times \widetilde{N}} \\
\beta=\left[\begin{array}{c}
\beta_{1}^{T} \\
\vdots \\
\beta_{\widetilde{N}}^{T}
\end{array}\right]_{\widetilde{N} \times m} \quad \text { and } T=\left[\begin{array}{c}
t_{1}^{T} \\
\vdots \\
t_{N}^{T}
\end{array}\right]_{N \times m}
\end{gathered}
$$

$\mathrm{H}$ is called the hidden layer output matrix of the network[14]; the ith column of $\mathrm{H}$ is the ith hidden node's output vector with respect to inputs $X_{1}, X_{2}, \ldots, X_{N}$ and the jth rowof $\mathrm{H}$ is the output vector of the hidden layer with respect to input $X_{j}$.

In real applications, the number of hidden nodes, $\widetilde{N}$, willalways be less than the number of training samples, $\mathrm{N}$, and,hence, the training error cannot be made exactly zero but can approach a nonzero training error $\in$. The hidden nodeparameters $a_{i}$ and $b_{i}$ (input weights and biases or centersand impact factors) of SLFNs need not be tuned duringtraining and may simply be assigned with random valuesaccording to any continuous sampling distribution, [9], and [10]. Equation (5) then becomes a linear system and the output weights $\beta$ are estimated as

$$
\hat{\beta}=H^{+} T
$$

Where $H^{+}$the Moore-Penrose is generalized inverse [14] of the hidden layer output matrix $\mathrm{H}$. The ELM algorithm which consists of only three steps, can then be summarized as ELM Algorithm: Given a training set

$\mathcal{N}=\left\{\left(X_{i}, t_{i}\right) \mid X_{i} \in R^{n}, t_{i} \in R^{m}, i=1, \ldots, N\right\}$, activation function $g(x)$, and hidden node number $\widetilde{N}$.

STEP 1: Assign random hidden nodes by randomly generating parameters $\left(a_{i}, b_{i}\right)$ according to any continuous sampling distribution, $i=1, \ldots, \widetilde{N}$.

STEP 2: Calculate the hidden layer output matrix $\mathrm{H}$.

STEP 3: Calculate the output weight $\beta$

$$
\beta=H^{+} T
$$




\section{Experimental Result}

The proposed framework results are carried out in MATLAB. The input for proposed work is collected from the MIT-BIH arrhythmia database, and it gives the classification output as Normal and Abnormal. Totally 30 patient records are collected from that database. In this 15 signals are used for testing and another 15 is used for training. The classification is done by three methods that are Back Propagation Neural Network (BPNN), Support Vector Machine (SVM) and Extreme Learning Machine (ELM).

In this paper classification performance are evaluated using some metrics that are Sensitivity, Positive Predictivity and Specificity. The following equations are used to calculated these metrics

$$
\begin{aligned}
& S e(\%)=\frac{T P}{T P+F N} \times 100 \\
& P P(\%)=\frac{T P}{T P+F P} \times 100 \\
& S p(\%)=\frac{T N}{T N+F P} \times 100
\end{aligned}
$$

In the above three equation TP denotes the number of true positive samples, FN indicates the number of false negative samples, TN denotes the number of true negative samples and FP indicates the number of false positive samples. These TP, TN, FP and FN are used for classification and it is defined as

FP: Normal class classifies as abnormal.

TP: Abnormal class classifies as abnormal.

FN: Abnormal classifies as normal.

TN: Normal class classifies as normal.

Then Overall classification accuracy is evaluated using below equation

$$
\text { Overall Accuracy }(\%)=\frac{\text { Correctly classified samples }}{\text { Total Number of samples }}
$$

Figure 2illustrates the preprocessing output, figure 2(a) shows the original image and 2(b) illustrates the noise reduction median filter. The original image is collected from the MIT-BIH arrhythmia database and each signal is digitized at 360 samples per second per channel with 11-bit resolution over a $10 \mathrm{mV}$ range. The preprocessing output of median filter is given as the input to the feature extraction. Feature extraction is used to detect the PQRS peak waveform and it is shown in figure 3. Peak detection is done by maximum and minimum value of the signal.

Table 1 provides the normal and abnormal records for patients beats collected from the MIT-BIH database. Table 2 and 3 provides the results such as accuracy, positive Predictivity, specificity and sensitivity for ECG recordings for various classifiers such as ELM, SVM and BPNN. [24]

Classification results are shown and given by a confusion matrix, through this confusion matrix accuracy, specificity, sensitivity and Positive Predictivity is produced. Totally 30 ECG signals are picked from the MIT BIH arrhythmia database, in this 15 signals are used for training and 15 signals are used testing. From the confusion matrix of TP, TN, FP and FN, accuracy is calculated and given the output of $94 \%$ for ELM, $73 \%$ for SVM and $64 \%$ for BPNN.[25]

\section{Conclusion}

This paper described about the classification and analysis of ECG signal through normal and abnormal beats.

This paper divide the process into pre-processing, feature extraction and classification. From the denoised result, median filter proved better result.

Classification is analysed through Back Propagation Neural Network, Support Vector Machine and Extreme Learning Machine. Classified the ECG signal as normal and abnormal classes and it is collected from the MIT-BIH arrhythmia database, 30 records are collected from this database, and split 15 for training and 15 for testing. PQRS features and QRS complex are extracted in this paper.

This extraction is useful for the classification of normal and abnormal beats.

The method achieves average detection accuracy of $89 \%$, a sensitivity of $60 \%$, and a positive Predictivity of $67 \%$. QRS complexes and different kinds of noise and artifacts contained in the ECG signals of the database are reduced using median filter. ELM classification achieves much higher detection rates than SVM and BPNN classification method.

Table 1: Normal and Abnormal Beats

\begin{tabular}{|c|c|}
\hline BEATS & Patient Record Number \\
\hline NORIIAL & $100-101-103-105-106-112-113-114-115-116-117-121-122-123-201-$ \\
\hline ABNORVAL & $104-108-109-111-118-119-124-200-203-207-208-210-212-214-217-$ \\
\hline
\end{tabular}

Table 2: Overall Accuracy of Proposed Methods

\begin{tabular}{|c|c|c|c|c|}
\hline Methods & Proposed Targets & Normal & Abnormal & \multirow{2}{*}{ Accuracy } \\
\hline \multirow{3}{*}{ ELM } & Normal class & 6 & 0 & \multirow{3}{*}{$94 \%$} \\
\cline { 2 - 4 } & Abnormal class & 1 & 8 & \\
\cline { 2 - 4 } & Total & 7 & 8 & \\
\hline \multirow{3}{*}{ SVM } & Normal class & 5 & 2 & \multirow{3}{*}{$73 \%$} \\
\cline { 2 - 4 } & Abnormal class & 2 & 6 & \\
\cline { 2 - 4 } BPNN & Total & 7 & 3 & \multirow{3}{*}{$64 \%$} \\
\hline & Normal class & 4 & 5 & \\
\hline & Abnormal class & 3 & 8 & \\
\hline & Total & 7 & & \\
\hline
\end{tabular}

Table 3: Performance Metrics of Proposed Methods

\begin{tabular}{|c|c|c|c|}
\hline Performance Metrics & ELM & SVM & BPNN \\
\hline Specificity & 89 & 60 & 60 \\
\hline Sensitivity & 60 & 60 & 60 \\
\hline Positive Predictivity & 67 & 62 & 57 \\
\hline
\end{tabular}

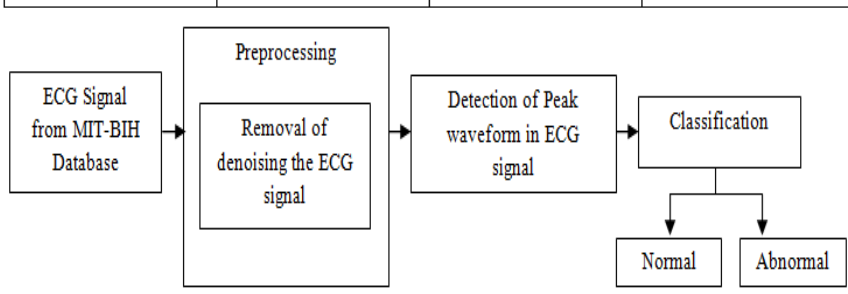

Figure 1: Block diagram of proposed framework

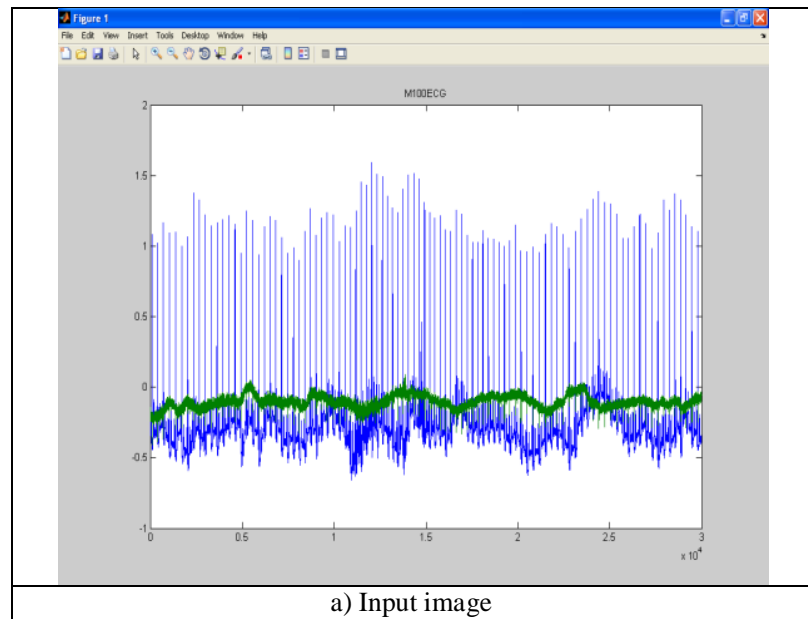




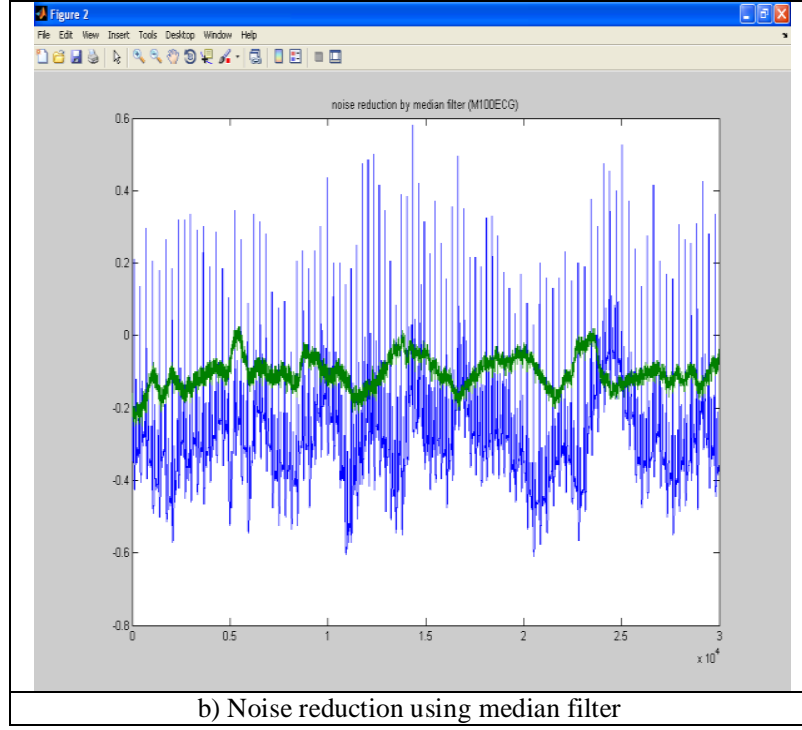

Figure 2: (a) Input image (b) Noise Reduction using Median filter

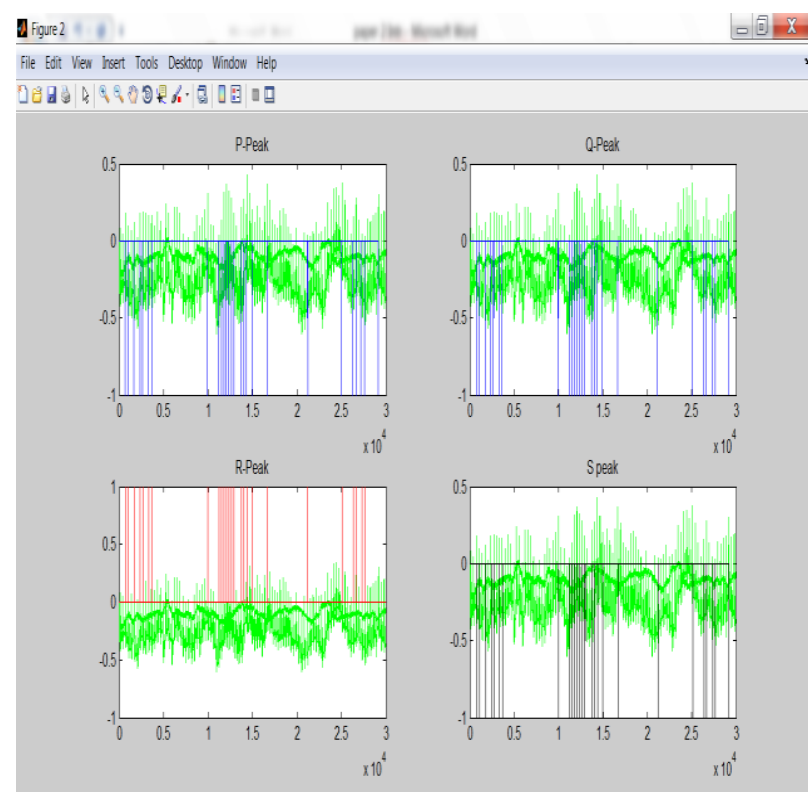

Figure 3: Feature extraction results of PQRS waveform peak detection

\section{References}

[1] Maglaveras N, Stamkopoulos T, Diamantaras K, Pappas C \& Strintzis M, "ECG pattern recognition and classification using nonlinear transformations and neural networks: a review", International journal of medical informatics, Vol.52, No.1-3, (1998), pp.191-208.

[2] De Chazal P, Celler BG \& Reilly RB, "Using wavelet coefficient for the classification of the electrocardiogram", IEEE 22nd Annual International Conference of the Engineering in Medicine and Biology Society, (2000), pp.64-67.

[3] Srinivasan N, Ge DF \& Krishnan SM, "Autoregressive modeling and classification of cardiac arrhythmias", IEEE 24th Annual Conference and the Annual Fall Meeting of the Biomedical Engineering Society, (2002), pp.1405-1406.

[4] Hussain H \& Fatt LL, "Efficient ECG signal classification using sparsely connected radial basis function neural network", 6th WSEAS International Conference on Circuits, Systems, Electronics, Control and Signal Processing, (2007), pp.412-416.

[5] Risk MR, Sobh JF \& Saul JP, "Beat detection and classification of ECG using self organizing maps", IEEE 19th Annual International Conference of the Engineering in Medicine and Biology Society, (1997), pp.89-91.
[6] Özbay Y, Ceylan R \& Karlik B, "Integration of type-2 fuzzy clustering and wavelet transform in a neural network based ECG classifier", Expert Systems with Applications, Vol.38, No.1, (2011), pp.1004-1010.

[7] Saxena SC, Kumar V \& Hamde ST, "Feature extraction from ECG signals using wavelet transforms for disease diagnostics", International Journal of Systems Science, Vol.33, No.13, (2002), pp.1073-1085.

[8] Foo SY, Stuart G, Harvey B \& Meyer-Baese A, "Neural networkbased EKG pattern recognition", Engineering Applications of Artificial Intelligence, Vol.15, No.3-4, (2002), pp.253-260.

[9] Sternickel K, "Automatic pattern recognition in ECG time series", Computer methods and programs in biomedicine, Vol.68, No.2, (2002), pp.109-115.

[10] Güler İ \& Übeylı ED, "ECG beat classifier designed by combined neural network model", Pattern recognition, Vol.38, No.2, (2005), pp.199-208.

[11] Übeyli ED, "Combining recurrent neural networks with eigenvector methods for classification of ECG beats", Digital Signal Processing, Vol.19, No.2, (2009), pp.320-329.

[12] Korürek M \& Doğan B, "ECG beat classification using particle swarm optimization and radial basis function neural network", Expert systems with Applications, Vol.37, No.12, (2010), pp.7563-7569.

[13] De Gaetano A, Panunzi S, Rinaldi F, Risi A \& Sciandrone M, "A patient adaptable ECG beat classifier based on neural networks", Applied Mathematics and Computation, Vol.213, No.1, (2009), pp.243-249.

[14] Choi S, Adnane M, Lee GJ, Jang H, Jiang Z \& Park HK, "Development of ECG beat segmentation method by combining low pass filter and irregular R-R interval checkup strategy", Expert Systems with Applications, Vol.37, No.7, (2010), pp.52085218.

[15] Ning X \& Selesnick IW, "ECG enhancement and QRS detection based on sparse derivatives", Biomedical Signal Processing and Control, Vol.8, No.6, (2013), pp.713-723.

[16] Poungponsri S \& Yu XH, "An adaptive filtering approach for electrocardiogram (ECG) signal noise reduction using neural networks", Neuro computing, Vol.117, (2013), pp.206-213.

[17] Yeh YC, Chiou CW \& Lin HJ, "Analyzing ECG for cardiac arrhythmia using cluster analysis", Expert Systems with Applications, Vol.39, No.1, (2012), pp.1000-1010.

[18] Saini I, Singh D \& Khosla A, "QRS detection using K-Neares Neighbor algorithm (KNN) and evaluation on standard ECG databases", Journal of advanced research, Vol.4, No.4, (2013), pp.331-344.

[19] Sadhukhan D \& Mitra M, "R-peak detection algorithm for ECG using double difference and RR interval processing", Procedia Technology, Vol.4, (2012), pp.873-877.

[20] Rai HM, Trivedi A \& Shukla S, "ECG signal processing for abnormalities detection using multi-resolution wavelet transform and Artificial Neural Network classifier", Measurement, Vol.46, No.9, (2013), pp.3238-3246.

[21] Mark R \& Moody G, "MIT-BIH arrhythmia database directory", Cambridge: Massachusetts Institute of Technology, (1988).

[22] Stallin S, Rajkumar P \& Rajendran K, "Reduction of Noises in ECG Signal by Various Filters", International Journal of Engineering Research \& Technology, Vol.3, No.1, (2014).

[23] Burges CJC, "A tutorial on support vector machines for pattern recognition”, Data mining and knowledge discovery, Vol.2, (1998), pp.955-971.

[24] A Mukanbetkaliyev, S Amandykova, Y Zhambayev, Z Duskaziyeva, A Alimbetova (2018). The aspects of legal regulation on staffing of procuratorial authorities of the Russian Federation and the Republic of Kazakhstan Opción, Año 33. 187-216.

[25] Villalobos Antúnez, JV (2017). Karl R. Popper, Heráclito y la invención del logos. Un contexto para la Filosofía de las Ciencias Sociales. Opción Vol. 33, Núm. 84. 5-11 